\title{
The association the patient-reported outcomes after periacetabular osteotomy with radiographic features: a short-term retrospective study
}

Yinuo Fan ${ }^{1 \dagger}$, Weifeng $\mathrm{Li}^{1 \dagger}$, Yunlong Wu' ${ }^{1}$, Ruoyu Li ${ }^{2}$, Guoju Hong ${ }^{4,5}$, Zhongfeng Li', Lixin Chen ${ }^{1}$, Hanjun Fang ${ }^{2}$, Chi Zhou ${ }^{2^{*}}$, Wei He ${ }^{3}$ and Zhenqiu Chen ${ }^{2^{*}}$

\begin{abstract}
Background: Bernese periacetabular osteotomy $(\mathrm{PAO})$ is an effective treatment for patients with developmental dysplasia of the hip (DDH). PAO has been widely used in China, but few follow-up outcomes have been reported in the international community. Moreover, the risk factors affecting patient-reported outcomes have not been discussed in recent studies. In this study, patient-reported outcomes after PAO were reported, and risk factors affecting patientreported outcomes were analyzed.

Methods: Patients who underwent PAO for DDH from January 2014 to January 2020 were selected as the study subjects, and 66 hips were included in the analysis after screening $(59$ patients, with an average follow-up time of 3.01 years). The Harris Hip Score (HHS) and International Hip Outcome Instrument-12 (iHOT-12) were used to assess hip function and patient quality of life. The changes of preoperative and latest follow-up HHSs less than 9 were defined as symptomatic hips, that is, an adverse outcome; otherwise, the score indicates preserved hips. Also, the changes of preoperative and latest follow-up iHOT-12 were defined as symptomatic hips and preserved hips. Multivariate logistic regression analysis was used to predict the risk factors influencing the patient-reported outcomes, and receiver operating characteristic (ROC) curve analysis was performed on the risk factors to determine their sensitivity, specificity and cutoff value.
\end{abstract}

Results: Clinical outcome analysis demonstrates marked improvements in patient-reported outcomes. The multivariate logistic regression analysis showed that when the postoperative LCEA was $>38^{\circ}$, adverse outcomes were much more likely. However, a Tönnis angle of $-10^{\circ}$ to $0^{\circ}$ was a protective factor. In addition, hips with fair or poor joint congruency were more likely to develop negative outcomes. The ROC curve analysis showed that the optimal thresholds for the LCEA and Tönnis angles used to predict outcomes after PAO were $38.2^{\circ}$ and $-9^{\circ}$, respectively. Based on the results of the ROC curve analysis, among hips with poor or fair joint congruency preoperatively treated by surgeons who obtained the improper postoperative LCEAs and Tönnis angles, bad patient-reported outcomes will most likely be obtained.

\footnotetext{
*Correspondence: chenzq2019@126.com;642899538@qq.com

${ }^{\dagger}$ Yinuo Fan and Weifeng Li contributed equally to this work.

${ }^{2}$ The Department of Orthopedics, The First Affiliated Hospital

of Guangzhou University of Chinese Medicine, Guangzhou 510405,

Guangdong Province, People's Republic of China

Full list of author information is available at the end of the article
}

(c) The Author(s) 2021. Open Access This article is licensed under a Creative Commons Attribution 4.0 International License, which permits use, sharing, adaptation, distribution and reproduction in any medium or format, as long as you give appropriate credit to the original author(s) and the source, provide a link to the Creative Commons licence, and indicate if changes were made. The images or other third party material in this article are included in the article's Creative Commons licence, unless indicated otherwise in a credit line to the material. If material is not included in the article's Creative Commons licence and your intended use is not permitted by statutory regulation or exceeds the permitted use, you will need to obtain permission directly from the copyright holder. To view a copy of this licence, visit http://creativecommons.org/licenses/by/4.0/. The Creative Commons Public Domain Dedication waiver (http://creativeco mmons.org/publicdomain/zero/1.0/) applies to the data made available in this article, unless otherwise stated in a credit line to the data. 
Conclusions: Our results demonstrate marked improvements in patient-reported outcomes. Among hips with preoperative excellent or good joint congruency treated by experienced surgeons who obtain the proper postoperative LCEA and Tönnis angles, good patient-reported outcomes can be expected.

Keywords: Bernese periacetabular osteotomy, Patient-reported outcomes, Multivariate logistic regression analysis, Receiver operating characteristic curve

\section{Introduction}

Developmental dysplasia of the hip (DDH) refers to inadequate coverage of the femoral head by the acetabulum due to abnormal structural development of the hip, which leads to subluxation or complete dislocation of the hip [1,2]. Periacetabular osteotomy (PAO), first used by Ganzs, is one of the most commonly used hip-conserving surgeries for DDH [3]. PAO not only corrects the lateral, anterior coverage, forward and backward tilt, and inward and outward displacement of the acetabulum but also ensures continuity of the posterior column of the pelvis and provides satisfactory correction of the acetabulum in DDH patients, thereby delaying hip osteoarthritis and total hip arthroplasty (THA), rendering it fully applicable to young patients [4].

This surgery has been widely used in many countries, and some follow-up results have confirmed the efficacy of PAO [5-17]. China was one of the countries to adopt PAO early, but there are few follow-up studies on PAO, making current PAO studies important. In addition, we found that short-term follow-ups after PAO mostly addressed patient-reported outcomes with no discussion of risk factors affecting patient-reported outcomes [6, $8-11,14,15]$. In contrast with these short-term followup results, our study not only performed an analysis of the impact of postoperative radiographic parameters on patient-reported outcomes but also performed ROC analysis on the postoperative radiographic parameters to determine their cutoffs.

Considering the current situation, this study retrospectively analyzed the data of DDH patients treated with PAO from 2014 to January 2020. The purpose of this study was to evaluate the short-term efficacy of PAO for $\mathrm{DDH}$, analyze the association the patient-reported outcomes after PAO with radiographic features, and propose measures to improve patient-reported outcomes.

\section{Materials and methods General information}

After approval by the Institutional Review Committee, patients who underwent PAO for DDH in the First Affiliated Hospital of Guangzhou University of Chinese Medicine from 2014 to January 2020 were selected as study subjects. The diagnosis of DDH was determined by highly qualified physicians through radiographic evidence and symptoms. The inclusion criteria were: (1) radiographic evaluation was lower than Tönnis grade 3; (2) younger than 55 years old and older than 18 years old; (3) complete follow-up data and follow-up time is at least 1 year; (4) all DDH patients who met these criteria underwent PAO. The exclusion criteria were as follows: (1) isolated acetabular retroversion, neuromuscular or connective-tissue disorder, Legg-Calve-Perthes disease, or DDH combined with other diseases, such as LeggCalve-Perthes disease or acetabular retroversion; (2) Tönnis grade 3 or other hip trauma; and (3) patients with no follow-up due to interrupted communication. The screening process of cases is shown in Fig. 1.

\section{Preoperative and postoperative examination}

Before PAO, the functional activity of the hip and quality of life of the patient were evaluated by Harris Hip Score (HHS) [18] and International Hip Outcome Tool12 (iHOT-12) [19], and a standard anteroposterior radiograph of the pelvis was routinely taken. After PAO, the HHS and iHOT-12 ratings were evaluated, and a standard anteroposterior radiograph of the pelvis was taken.

\section{Outcome measures}

The HHS and iHOT-12 were used to evaluate the joint activity, pain, and quality of life in preoperative and postoperative patients with PAO. HHSs can be divided into four grades: excellent $(\geq 90)$, good (80-89), fair (70-79), and poor $(<70)$ and include measures of pain, function, joint movement, limb deformity, etc. In this study, adverse outcomes were defined through HHSs. According to previous study [20], it has been found that the minimal clinically important difference (MCID) is 7-9 for the HHS and 13 for the iHOT-12 [21]. Thus, the changes of preoperative and latest follow-up HHSs less than 9 were defined as symptomatic hips, that is, an adverse outcome; otherwise, the score indicates preserved hips. Also, the changes of preoperative and latest follow-up iHOT-12 were defined as symptomatic hips and preserved hips. From August to September 2020, all included patients were administered a questionnaire.

The lateral center-to-edge angle (LCEA), acetabular coverage ratio, sharp angle and Tönnis angle were used to evaluate the radiographic correction degree during the PAO. LCEA, acetabular coverage ratio, sharp angle and 


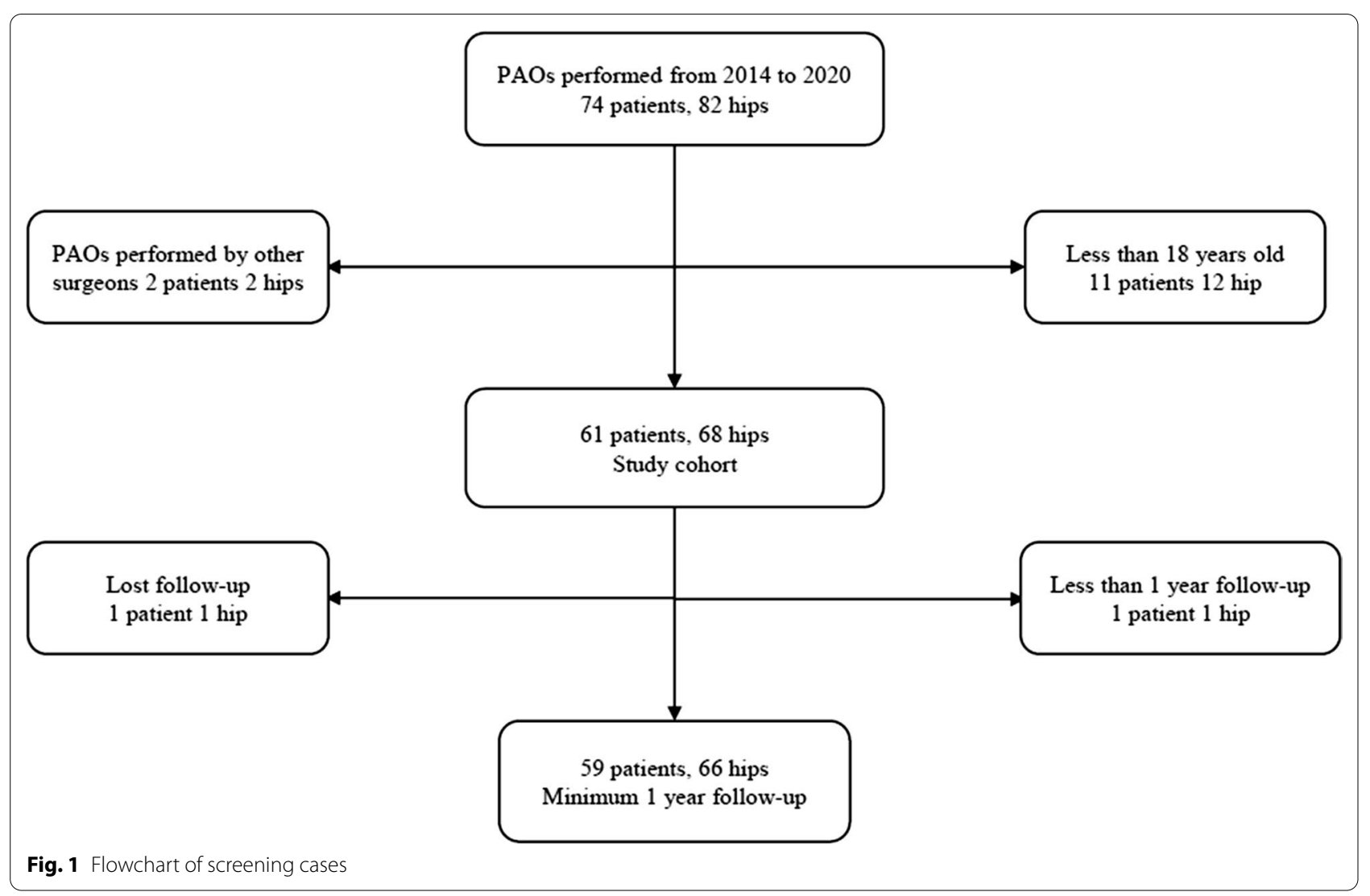

Tönnis angle were measured by Digimizer software (version 5.4.6) before and after the PAO. DDH is generally believed to exist with an LCEA $<20^{\circ}[22,23]$, acetabular coverage ratio $<75 \%$ [24], sharp angle $>40^{\circ}$ [25] and Tönnis angle $>10^{\circ}[26]$. In addition, according to previous studies [22, 23], the preoperative LCEA was graded as follows: LCEA $<5^{\circ}, 5^{\circ}<$ LCEA $<20^{\circ}$, and LCEA $>20^{\circ}$. According to Wells et al. [17], a postoperative LCEA was graded as $20^{\circ}<\mathrm{LCEA}<38^{\circ}$ and LCEA $>38^{\circ}$. There is no uniform standard for the range of correction for the Tönnis angle after PAO. According to the experience of the surgeons involved with this study, the postoperative Tönnis angle was within the appropriate range at $0 \pm 10^{\circ}$. Therefore, we graded the postoperative Tönnis angle as $0<$ Tönnis angle $<10^{\circ},-10^{\circ}<$ Tönnis angle $<0$ and Tönnis angle $<-10^{\circ}$. All of these indicators were measured and observed by three authors, and the final results were averaged.

The Tönnis classification of hip osteoarthritis [27, 28] was divided into four grades: 0 (no signs of osteoarthritis), 1 (slight narrowing of the joint space, slight lipping at the joint margin, and slight sclerosis of the femoral head or acetabulum), 2 (small cysts in the femoral head or acetabulum, moderate narrowing of the joint space, and moderate loss of sphericity of the femoral head), and 3 (large cysts, severe narrowing or obliteration of the joint space, severe deformity of the femoral head, and avascular necrosis). Joint congruency [29] was described by excellent (radii of curvature of the acetabulum and femoral head identical and joint space maintained), good (curvature of the femoral head and acetabulum not identical, but joint space preserved), fair (joint space partially narrowed), and poor (loss of joint space). Hips with excellent or good joint congruency were considered an acceptable outcome.

\section{Surgical technique Incision and approach}

In the supine position, the anterolateral S-P incision approach of the hip joint is taken. The skin and subcutaneous are cut in sequence. The lateral femoral cutaneous nerve is exposed and protected along the way. Along the sartorius, rectus femoris, and tensor fascia lata muscles, the surgeon strips the iliac muscle of the medial iliac bone to the quadrilateral and then performs an osteotomy of approximately $2 \times 1 \mathrm{~cm}$ in the anterior superior iliac spine. Finally, the surrounding muscles were gradually stripped to reveal the ischial notch, ischial spine and ischial minor notch. 


\section{Osteotomy}

Under c-arm fluoroscopy, the surgeon uses a crescent knife to perform incomplete osteotomy (about $1 / 2$ to $1 / 3$ ) of the proximal ischial branch close to the acetabular groove. After that, the surgeon performed a complete osteotomy of the proximal medial tuberosity of the pubis, then peeled off part of the periosteum of the outer plate of the iliac bone, and performed a vertical osteotomy at the anterior inferior iliac spine to the arcuate line about $1.5 \mathrm{~cm}$. Finally, the osteotomy of the medial quadrilateral, ischial spine, and ischial branch was exposed, and a quadrilateral osteotomy was performed at a distance of about $1.5 \mathrm{cn}$ behind the ischial notch.

\section{Fix the osteotomy site and close the incision}

The surgeon uses 3 bone screws to fix the anterior inferior iliac spine osteotomy site and 2 fix the anterior superior iliac spine osteotomy block. After the surgeon determined that the passive movement of the hip joint was good, the osteotomy angle and the internal fixation under the c-arm fluoroscopy were satisfactory, and then, the incision was closed layer by layer.

\section{Postoperative care and outcome evaluation}

Postoperatively, the surgical hip was kept abducted in a neutral position for two weeks. The patients were encouraged to actively exercise the limb and were instructed to undergo quadriceps femoris and gastrocnemius muscle isometric contraction training and ankle pump exercises. Unilateral DDH patients could get out of bed early to practice walking with two crutches, while bilateral DDH patients were told to walk with help for half a year after the surgery. The patient can bear partial weight 3 months after the operation, and half a year after the operation, consider whether to bear the weight completely according to the healing of the fracture.

Postoperative follow-up was carried out at three days, one week, three months, six months and 12 months. At each appointment, anteroposterior and lateral $\mathrm{X}$-rays were taken for radiographic evaluation. Clinical outcomes were assessed by comparing the HHS and iHOT-12 before surgery and at the latest follow-up appointment.

\section{Statistical analysis}

Continuous variables are expressed as the means and standard deviations, and categorical variables are expressed as numbers and percentages. When preoperative and postoperative radiographic parameters and patient-reported outcomes were analyzed, a t-test was used when they conformed to a normal distribution; the Wilcoxon signed-rank test was used when they did not conform to a normal distribution. Variance inflation factor (VIF) was used to determine whether there is multicollinearity between various indicators. Multivariate logistic regression analysis was used to conduct statistical analysis on the included parameters to predict the risk factors influencing the outcome, and receiver operating characteristic (ROC) curve analysis was performed on the risk factors to determine their sensitivity, specificity and cutoff value. $p<0.05$ was considered statistically significant, and all data were statistically analyzed with SPSS Software version 23.0 (IBM).

\section{Results}

A total of 59 patients (17 male and 42 female) were included in the analysis. The mean follow-up time was $3.01 \pm 1.19$ years ( $1-6$ years). The mean age and BMI were $33.61 \pm 9.31$ years old (range, from 18 to 54 years old) and $22.37 \pm 1.84$, respectively (Table 1 ).

\section{Patient-reported outcomes}

The changes in patient-reported outcomes were significantly different between the final follow-up and the preoperative results. The patients reported a mean improvement from a $61.00 \pm 16.16$ preoperatively to a $80.65 \pm 7.14$ at the last follow-up $(p<0.001)$ on the HHS scale and from $60.94 \pm 22.41$ to $87.11 \pm 19.98$ $(p<0.001)$ on the iHOT-12 scale (Table 1$)$. In addition, the comparison between the HSS of the preserved hip group $(86.02 \pm 3.29)$ and the symptomatic hip group

Table 1 Patient characteristics

\begin{tabular}{|c|c|}
\hline \multicolumn{2}{|l|}{ Characteristic } \\
\hline Number of patients (hips) & $59(66)$ \\
\hline $\mathrm{Age}^{\dagger}$ (year) & $33.61 \pm 9.31(18-54)$ \\
\hline Females (no. [\%]) & $42(71.2 \%)$ \\
\hline Time to latest follow-up $(y r)^{\dagger}$ & $3.01 \pm 1.19(1-6)$ \\
\hline Height $^{\ddagger}(m)$ & $1.60 \pm 0.07$ \\
\hline Weight $^{\ddagger}(\mathrm{kg})$ & $57.79 \pm 7.87$ \\
\hline Body Mass Index, $\mathrm{BMI}^{\ddagger}\left(\mathrm{kg} / \mathrm{m}^{2}\right)$ & $22.37 \pm 1.84$ \\
\hline \multicolumn{2}{|l|}{$\mathrm{HHS}$} \\
\hline Preoperative $^{\ddagger}$ & $61.00 \pm 16.16$ \\
\hline Postoperative $^{\ddagger}$ & $80.65 \pm 7.14$ \\
\hline$P$ value & $<0.001$ \\
\hline \multicolumn{2}{|l|}{ iHOT-12 } \\
\hline Preoperative ${ }^{\ddagger}$ & $60.94 \pm 22.41$ \\
\hline Postoperative $^{\ddagger}$ & $87.11 \pm 19.98$ \\
\hline P value & $<0.001$ \\
\hline
\end{tabular}

${ }^{\dagger}$ The values are given as the mean and the standard deviation, with the range in parentheses

₹ The values are given as the mean and the standard deviation 


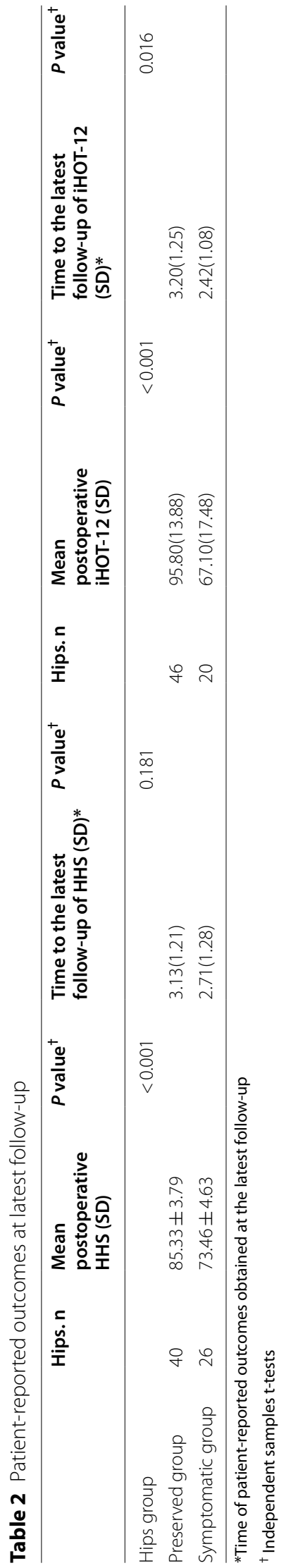


$(74.06 \pm 5.99)$ was statistically significant $(p<0.001)$. The comparison between the iHOT-12 of the preserved hip group and the symptomatic hip group was also statistically significant $(p<0.001)$ (Table 2$)$. By comparing the follow-up times of the preserved hip group and those of the symptomatic hip group, we found that the difference was not statistically significant (Table 2), which indicated that the difference in the follow-up time between the two groups did not lead to bias in the HHS-based results. However, the follow-up times of iHOT-12 got adverse result.

\section{Radiographic parameters outcomes}

The progression of 66 hips in terms of Tönnis grade is shown in Fig. 2. After PAO, the hips were classified according to the changes of HHS, and a total of 40 preserved hips and 26 symptomatic hips were documented. 46 preserved hips and 20 symptomatic hips were analysed in the iHOT12-based results. Whether it was evaluated by HHS or iHOT-12, the statistical results showed that postoperative LCEA, Tönnis angle and preoperative poor or fair joint congruency were all statistically significant (Table 3).

The radiographic parameters were greatly changed before and after PAO. The acetabular coverage ratio improved from $0.58 \pm 0.14$ to $0.90 \pm 0.09(p<0.001)$, the sharp angle improved from $47.78 \pm 4.33$ to $33.86 \pm 4.34$ $(p<0.001)$, the LCEA improved from $9.02 \pm 13.08$ to $38.02 \pm 8.28(p<0.001)$, and the Tönnis angle improved from $22.82 \pm 9.45$ to $0.07 \pm 7.13(p<0.001$, Table 4$)$.

Risk factors were predicted based on multivariate logistic regression analysis. This study included age, sex, BMI, preoperative and postoperative LCEA, acetabular coverage ratio, sharp angle, preoperative Tönnis grades, Tönnis angle and joint congruency in the risk factor analysis. The results showed that the LCEA (Fig. 3), Tönnis angle and preoperative joint frequency had a significant influence on the outcome, and the remaining factors were not significantly different. The VIFs of LCEA, Tönnis angle and preoperative joint frequency were all 1.79, 1.97 and 1.27 in the HHS-based results and iHOT-12-based results, respectively. The results of The VIFs showed that there was no strong multicollinearity between these indicators. When the postoperative LCEA was $>38^{\circ}$, the risks of an adverse outcome were 16.093-fold higher (odds ratio [OR]: 16.093; 95\% CI 1.696-102.788; $p=0.003$; HHS; Table 5) and 10.854-fold higher (odds ratio [OR]: 10.854; 95\% CI 2.520-69.475; $p=0.012$; iHOT12; Table 5). A Tönnis angle of $10^{\circ}-0^{\circ}$ was a protective factor (odds ratio [OR]: 0.083 ; 95\% CI $0.012-0.554$ ) in the HHS-based results, but not in the iHOT-12-based results. In addition, hips with fair or poor joint congruency were 4.793 times (odds ratio [OR]: 4.793; 95\% CI 1.137-20.214;
HHS; $p=0.004$ ) and 8.960 times (odds ratio [OR]: 8.960; 95\% CI 1.892-42.442; iHOT12; $p=0.006$ ) more likely to develop negative outcomes.

The ROC analysis revealed that the cutoff points for LCEA and Tönnis angle were 38.2 (sensitivity $=77 \%$, specificity $=70 \%, \mathrm{AUC}=71.1 \%$, log-rank test: $p<0.0001$, HHS/ sensitivity $=85 \%$, specificity $=67 \%$, AUC $=75.0 \%$, log-rank test: $p<0.0001$, iHOT-12) and -9 (sensitivity $=69 \%$, specificity $=95 \%, A U C=62.8 \%$, log-rank test: $p<0.0001$, HHS / sensitivity $=65 \%$, specificity $=94 \%$, AUC $=68.3 \%$, log-rank test: $p<0.0001$, iHOT-12) in the HHS-based results and iHOT-12-based results, respectively. The result showed that preoperative joint congruency (sensitivity $=69.2 \%$, specificity $=72.5 \%$, AUC $=70.9 \%$, log-rank test: $p<0.0001$, HHS / sensitivity $=71.7 \%, \quad$ specificity $=94 \%, \quad \mathrm{AUC}=75.9 \%, \quad$ log-rank test: $p<0.0001$, iHOT-12) (Table 6).

\section{Complication}

During the follow-up, there were infections in two hips, nonunion of fracture in one hip, minor nerve damage in 10 hips.

\section{Discussion}

DDH has a high incidence in China, with an incidence of $2.9 \%$ in Taiwan [30]. If left untreated, joint wear gradually increases and eventually a THA is required [31]. Therefore, hip preservation is a good choice for young patients with $\mathrm{DDH}$. The purpose of hip preservation is to alleviate pain symptoms, slow the progression of osteoarthritis, and delay or even prevent the need for a THA by correcting the hip malformations. Compared with THA, PAO is an ideal choice for young people with symptomatic DDH $[32,33]$. Current follow-up results confirmed the efficacy of PAO [5-17]. China was one of the countries to adopt PAO early, but there are few follow-up studies on PAO in the international community. Therefore, we followed patients with DDH after PAO to observe the short-term efficacy of PAO and to predict the factors that affect patient-reported outcomes.

Our study was one of the largest short-term followup studies analyzing patient-reported outcomes after PAO performed to alleviate symptomatic DDH. We analyzed the factors that influenced patient-reported outcomes after PAO. In this retrospective study, we found that patients who underwent PAO in our study had good postoperative radiographic parameters and outcome improvement as indicated by patient reports. Additionally, the patients' progression of Tönnis grade was not obvious. Our data suggest that while hips with fair or poor joint congruency preoperatively and an oversized postoperative LCEA were correlated with unsatisfactory patient-reported outcomes, the proper 


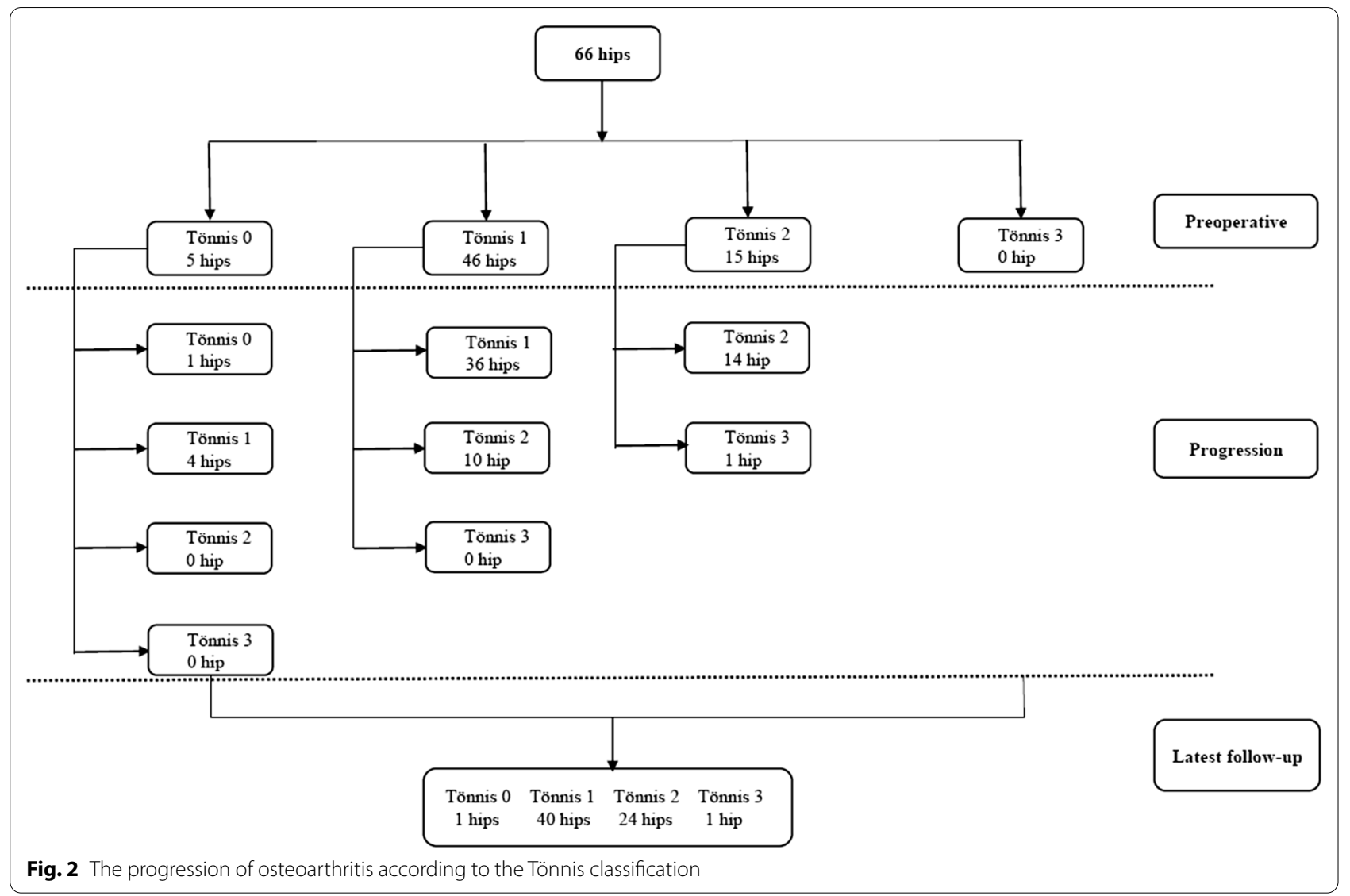

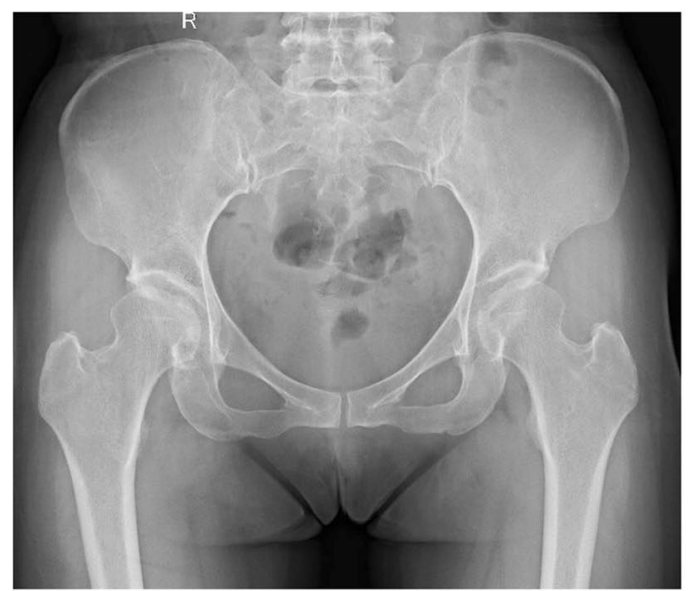

a

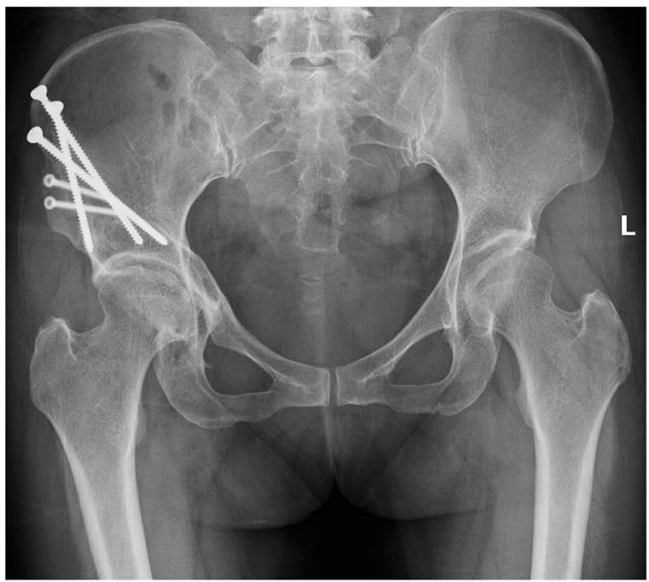

b

Fig. 3 Female, 46 years. a Preoperative X-ray. b Postoperative X-ray. The preoperative LCEA was $24^{\circ}$, and the postoperative LCEA was adjusted to $55^{\circ}$. The preoperative HHS (iHOT-12) was 67 (58), and the postoperative HHS was 73 (70). The MCID of HHS (iHOT-12) was 6 (12)

postoperative Tönnis angle was a positive factor for good patient-reported outcomes in the HHS-based results. When the LCEA and Tönnis angle was $38.2^{\circ}$ and $-9^{\circ}$, respectively, these are two critical values affected patient-reported outcomes. However, the Tönnis angle was not a related factor for clinical outcome in the Ihot12-based results. We guess that HHS mainly evaluates joint function, while iHOT-12 mainly evaluates 
Table 3 Patient characteristics of cohort

\begin{tabular}{|c|c|c|c|c|c|c|}
\hline \multirow[t]{2}{*}{ Radiographic parameters } & \multicolumn{2}{|l|}{ No. (HHS) } & \multirow[t]{2}{*}{$P$ Value $^{\dagger}$} & \multicolumn{2}{|l|}{ No. (iHOT12) } & \multirow[t]{2}{*}{$P$ Value } \\
\hline & $\begin{array}{l}\text { Preserved } \\
\text { ( } n=40 \text { hips) }\end{array}$ & $\begin{array}{l}\text { Symptomatic } \\
\text { ( } n=26 \text { hips) }\end{array}$ & & $\begin{array}{l}\text { Preserved } \\
\text { ( } n=46 \text { hips) }\end{array}$ & $\begin{array}{l}\text { Symptomatic } \\
\text { ( } n=20 \text { hips) }\end{array}$ & \\
\hline \multicolumn{7}{|c|}{ Preoperative radiographic parameters } \\
\hline Acetabular coverage ratio $<75 \%$ & $40(100 \%)$ & $23(88 \%)$ & 0.111 & $46(100 \%)$ & $17(85 \%)$ & 0.025 \\
\hline Sharp angle $>40^{\circ}$ & $39(98 \%)$ & $25(96 \%)$ & 1.000 & $44(96 \%)$ & $20(100 \%)$ & 1.000 \\
\hline LCEA & & & 0.108 & & & 0.128 \\
\hline$<5^{\circ}$ & $15(38 \%)$ & $5(19 \%)$ & & $16(35 \%)$ & $4(20 \%)$ & \\
\hline $5^{\circ}-20^{\circ}$ & $21(53 \%)$ & $14(54 \%)$ & & $25(54 \%)$ & $10(50 \%)$ & \\
\hline$>20^{\circ}$ & $4(10 \%)$ & $7(27 \%)$ & & $5(11 \%)$ & $6(30 \%)$ & \\
\hline Tönnis angle $>10^{\circ}$ & $39(98 \%)$ & $25(96 \%)$ & 1.000 & $45(98 \%)$ & 19 (95\%) & 0.517 \\
\hline Joint congruency poor or fair & $11(28 \%)$ & $18(69 \%)$ & 0.001 & $13(28 \%)$ & $16(80 \%)$ & 0.000 \\
\hline \multicolumn{7}{|c|}{ Postoperative radiographic parameters } \\
\hline Acetabular coverage ratio $>75 \%$ & $40(100 \%)$ & $26(100 \%)$ & 1.000 & $46(100 \%)$ & $20(100 \%)$ & 1.000 \\
\hline Sharp angle $<40^{\circ}$ & $36(90 \%)$ & $25(96 \%)$ & 0.655 & $42(91 \%)$ & $19(95 \%)$ & 0.988 \\
\hline LCEA & & & 0.000 & & & 0.001 \\
\hline$<20^{\circ}$ & 0 & $1(4 \%)$ & & $1(2 \%)$ & 0 & \\
\hline $20^{\circ}-38^{\circ}$ & 27 (68\%) & $5(19 \%)$ & & $29(63 \%)$ & $3(15 \%)$ & \\
\hline$>38^{\circ}$ & $13(33 \%)$ & $20(77 \%)$ & & $16(35 \%)$ & 17 (85\%) & \\
\hline Tönnis angle & & & 0.012 & & & 0.011 \\
\hline $0^{\circ}-10^{\circ}$ & $25(63 \%)$ & $13(50 \%)$ & & $30(65 \%)$ & $8(40 \%)$ & \\
\hline$-10^{\circ}-0^{\circ}$ & $14(35 \%)$ & $6(23 \%)$ & & $14(30 \%)$ & $6(30 \%)$ & \\
\hline$<-10^{\circ}$ & $1(3 \%)$ & $7(27 \%)$ & & $2(4 \%)$ & $6(30 \%)$ & \\
\hline Joint congruency poor or fair & $7(18 \%)$ & $8(31 \%)$ & 0.209 & $9(20 \%)$ & $6(30 \%)$ & 0.542 \\
\hline
\end{tabular}

${ }^{\dagger}$ Based on univariable comparisons between preserved and symptomatic hips

Table 4 Radiographic correction

\begin{tabular}{lccc}
\hline Characteristic & \multicolumn{2}{c}{ Mean (SD) } & Pvalue $^{\boldsymbol{*}}$ \\
\cline { 2 - 3 } & Preoperative & Postoperative & \\
\hline Acetabular coverage ratio & $0.58 \pm 0.14$ & $0.90 \pm 0.09$ & $<0.001$ \\
Sharp angle $\left(^{\circ}\right)$ & $47.78 \pm 4.33$ & $33.86 \pm 4.34$ & $<0.001$ \\
LCEA $\left(^{\circ}\right)$ & $9.02 \pm 13.08$ & $38.02 \pm 8.28$ & $<0.001$ \\
Tönnis angle $\left(^{\circ}\right)$ & $22.82 \pm 9.45$ & $0.07 \pm 7.13$ & $<0.001$ \\
\hline
\end{tabular}

*The values are given as the mean and the standard deviation

${ }^{\dagger}$ Based on univariable comparisons between preoperative and postoperative outcome

psychological factors, which leads to different results. Based on the results of the nomograms, among hips with poor or fair joint congruency preoperatively treated by surgeons who obtained the improper postoperative LCEAs and Tönnis angles, bad patient-reported outcomes will most likely be obtained.

In a study of 123 hips followed for a mean of 4.3 years after PAO, Trumble et al. [14] reported that 102 hips were preserved, 7 hips required a THA, and 6 subsequent intertrochanteric osteotomies were performed. The patients reported a mean improvement from 65 points preoperatively to 89 points at the latest follow-up, based on HHSs. The latest follow-up radiographic severity of osteoarthrosis, assessed according to Tönnis grade, progressed in only 6 hips. Although this study on PAO revealed good outcomes, only a simple follow-up was performed, and risk factors were not explored in depth. In another prospective, multicenter cohort of 391 hips followed for a mean of 2.6 years after PAO, Clohisy et al. [6] reported that age, sex and BMI were predictive factors of certain outcome measures. This study suggested that the strongest predictors of successful outcomes were female sex, increased age, and a high BMI. In addition, pain, hip function, and quality of life improved after PAO. In our study, 66 hips after PAO performed to alleviate DDH were followed for a mean of 3.01 years. This study showed that sex, age and BMI had no effect on the outcome measures, which may be related to it being a singlecenter study and including only a limited number of hips.

Wells et al. [16] and Matheney et al. [34] reported that poor or fair preoperative joint congruency is a risk factor for failure, which was consistent with our findings. Another study by Wells et al. [17] analyzed the outcomes of 154 hips followed for an average of 10.3 years and found that excessive postoperative femoral head 
Table 5 The multivariate logistic regression analysis

\begin{tabular}{|c|c|c|c|c|}
\hline \multirow[t]{2}{*}{ Variable } & \multicolumn{2}{|l|}{$\mathrm{HHS}^{\ddagger}$} & \multicolumn{2}{|l|}{ iHOT12 ${ }^{\ddagger}$} \\
\hline & OR $(95 \% \mathrm{Cl})$ & $P$ value & OR $(95 \% \mathrm{Cl})$ & $P$ value \\
\hline Postoperative LCEA* & & 0.013 & & 0.042 \\
\hline$<20^{\circ}$ & & 1.000 & & 1.000 \\
\hline$>38^{\circ}$ & $16.093(2.520-102.788)$ & 0.003 & $10.854(1.696-69.475)$ & 0.012 \\
\hline Postoperative Tönnis angle ${ }^{\dagger}$ & & 0.023 & & 0.17 \\
\hline$<-10^{\circ}$ & & 0.925 & & \\
\hline$-10^{\circ}-0^{\circ}$ & $0.087(0.012-0.656)$ & 0.018 & & \\
\hline $\begin{array}{l}\text { Preoperative joint congruency poor or } \\
\text { fair }\end{array}$ & $4.793(1.137-20.214)$ & 0.004 & $8.960(1.892-42.442)$ & 0.006 \\
\hline
\end{tabular}

*The statistical results of $<20^{\circ}$ and $>38^{\circ}$ in postoperative LCEA were compared with $20^{\circ}-38^{\circ}$

${ }^{\dagger}$ The statistical results of $<-10^{\circ}$ and $-10^{\circ}-0^{\circ}$ in postoperative LCEA were compared with $0^{\circ}-10^{\circ}$

${ }^{\ddagger}$ Using $\mathrm{HHS}$ and $\mathrm{iHOT} 12$ as the outcome indicators, respectively

Table 6 Prognostic values of postoperative Tönnis angle, LCEA and

\begin{tabular}{|c|c|c|c|c|}
\hline & Cutoff value & AUC (\%) & Specificity/sensitivity & Youden's index \\
\hline \multicolumn{5}{|l|}{$\mathrm{HHS}^{*}$} \\
\hline LCEA & 38.2 & 71.1 & $70 \% / 77 \%$ & 0.47 \\
\hline Tönnis angle & -9 & 62.8 & $95 \% / 69 \%$ & 0.64 \\
\hline Joint congruency & - & 70.9 & $72.5 \% / 69.2 \%$ & 0.417 \\
\hline \multicolumn{5}{|l|}{$\mathrm{iHOT}_{1}{ }^{\dagger}$} \\
\hline LCEA & 38.2 & 75.0 & $67 \% / 85 \%$ & 0.52 \\
\hline Tönnis angle & -9 & 68.3 & $94 \% / 65 \%$ & 0.59 \\
\hline Joint congruency & - & 75.9 & $94 \% / 71.7 \%$ & 0.657 \\
\hline
\end{tabular}

*ROC analysis with HHS as the outcome indicator

${ }^{\dagger} \mathrm{ROC}$ analysis with iHOT12 as the outcome indicator

‡ Joint congruency was divided into two variables, namely joint congruency poor or fair and joint congruency excellent or good. The cutoff cannot be calculated for binary variables

coverage $\left(\right.$ LCEA $\left.>38^{\circ}\right)$ was a predictor of failure. Additionally, Albers et al. [5] reported 165 hips after PAO and found that improper acetabular reorientation may accelerate osteoarthritis progression in patients with DDH, and proper acetabular reorientation without introducing femoroacetabular impingement (FAI) improved hip survivorship. To our knowledge, while there have been no other studies to indicate how radiographic parameters can be corrected to obtain favorable outcomes, the current studies showed that an LCEA of $>38^{\circ}$ may increase the risk of adverse outcomes. We speculate that the cause of the adverse outcomes may be associated with excessive acetabular coverage, which increases the chance of secondary FAI [5, 17]. Additionally, the correction of the postoperative Tönnis angle after PAO was unclear. According to the experience of the surgeon, a Tönnis angle of $0 \pm 10^{\circ}$ is in an acceptable range and was achieved in these surgeries. Our multivariate logistic regression analysis found that a Tönnis angle of $10^{\circ}-0$ was a protective factor. Furthermore, considering the results of ROC analysis, our study suggested that a Tönnis angle of $-9^{\circ}-0$ may result in better outcomes (Table 5). Not only there is no previous study to support this result, but different surgeons may have different understandings of the correction of the Tönnis angle; therefore, larger multicenter, prospective studies are needed to verify and explain why a postoperative Tönnis angle of $-9^{\circ}-0$ is a predictor of successful outcomes.

Our study had some limitations. First, compared with prospective and multicenter studies, the level of evidence in this retrospective and single-center studies is insufficient. All patients underwent surgery by experienced PAO surgeons, which may limit the generalizability of this study. Second, we included 7 patients for analysis who underwent bilateral PAO, which may have affected the results. Third, patients were subjectively selected for inclusion in a retrospective study, which can lead to bias. While we cannot eliminate possible selection bias, strict inclusion criteria can help with drawing significant conclusions. Forth, we only have the parameters of 
anteroposterior radiographs, but not the relevant parameters of frog-leg and false-profile radiographs. Last, we did not study other factors that influence clinical outcomes, such as Merle d'Aubigné-Postel score, preoperative limp and decreased preoperative internal rotation discovered by Lerch et al. [35] and delayed gadoliniumenhanced MRI of cartilage (dGEMRIC) found by Schmaranzer et al. [36].

Considering these limitations, our study presents novel findings. Short-term follow-up results in the past were mostly reports of patient-reported outcomes, and few studies included baseline or preoperative radiographic parameters in the multinomial logistic regression analysis for the prediction of risk factors. In contrast with these short-term follow-up results, our study not only performed an analysis of the impact of postoperative radiographic parameters on patient-reported outcomes but also performed ROC analysis on the postoperative LCEAs and Tönnis angles to determine their cutoffs.

\section{Conclusion}

Taken together, our results demonstrate marked improvements in patient-reported outcomes after PAO. Among hips with excellent or good joint congruency preoperatively treated by experienced surgeons who obtained the proper postoperative LCEAs and Tönnis angles, good patient-reported outcomes can be expected; the early symptomatic hip rates were low. Continued expansion and follow-up of this study will provide a higher level of clinical evidence to further determine how to improve patient-reported outcomes of PAO. Future studies should pay attention to comparing more postoperative radiographic parameters between asymptomatic and symptomatic hips to determine whether these parameters can be used as factors for predicting failure and as a reference value for PAO.

\section{Abbreviations}

PAO: Bernese periacetabular osteotomy; DDH: Developmental dysplasia of the hip; HHS: Harris hip score; iHOT-12: International Hip Outcome Instrument-12; ROC: Receiver operating characteristic; THA: Total hip replacement; LCEA: Lateral center-to-edge angle; FAl: Femoroacetabular impingement; dGEMRIC Delayed gadolinium-enhanced MRI of cartilage; VIF: Variance inflation factor.

\section{Authors' contributions}

Conceptualization: Yinuo Fan and Zhenqiu Chen. Literature search: Yinuo Fan, Weifeng Li and Guoju Hong. Data extraction and quality assessment: Weifeng Li and Yunlong Wu. Software: Weifeng Li, Lixin Chen and Yunlong Wu. Formal analysis: Yinuo Fan, Guoju Hong, and Hanjun Fang. Validation: Chi Zhou, Wei He and Zhenqiu Chen. Writing: Yinuo Fan. All authors read and approved the final manuscript.

\section{Funding}

This work was supported by High-level hospital construction project (The First Affiliated Hospital of Guangzhou University of Chinese Medicine, Grant No.: 211020030705) and Research Project of Innovating to Strengthen (The First Affiliated Hospital of Guangzhou University of Chinese Medicine, Grant
No.: 2019l|T06) in the form of covering the consultation fees of data statistical analysis.

\section{Availability of data and materials}

The datasets used or analysed during the current study are available from the corresponding author on reasonable request.

\section{Declarations}

\section{Ethics approval and consent to participate}

The institutional review board of the first affiliated hospital of Guangzhou University of Chinese Medicine approved this study. All methods were carried out in accordance with relevant guidelines and regulations. Informed consent was obtained from all subjects.

\section{Consent to participate}

Not applicable.

\section{Consent to publication}

All authors have seen the manuscript and approved it to submit to your journal.

\section{Competing interests}

The authors declare that they have to no competing interests.

\section{Author details}

${ }^{1}$ The First Clinical Medical College, Guangzhou University of Chinese Medicine, 12 Jichang Road, Baiyun District, Guangzhou 510405, Guangdong Province, People's Republic of China. ${ }^{2}$ The Department of Orthopedics, The First Affiliated Hospital of Guangzhou University of Chinese Medicine, Guangzhou 510405, Guangdong Province, People's Republic of China. ${ }^{3}$ Department of Joint Diseases, The Third Affiliated Hospital of Guangzhou University of Chinese Medicine, NO. 261 Longxi Road, Liwan District, Guangzhou, Guangdong Province, People's Republic of China. ${ }^{4}$ Candidate, Research Fellow, Division of Orthopaedic Surgery, The University of Alberta, Edmonton, Canada. ${ }^{5}$ Institute of Orthopedics, Guangzhou University of Chinese Medicine, Guangzhou, People's Republic of China.

Received: 22 July 2021 Accepted: 21 November 2021

Published online: 19 December 2021

\section{References}

1. MacKenzie IG, Wilson JG. Problems encountered in the early diagnosis and management of congenital dislocation of the hip. J Bone Joint Surg Br. 1981;63-B(1):38-42.

2. Dunn PM, Evans RE, Thearle MJ, Griffiths HE, Witherow PJ. Congenital dislocation of the hip: early and late diagnosis and management compared. Arch Dis Child. 1985;60(5):407-14.

3. Ganz R, Klaue K, Vinh TS, Mast JW. A new periacetabular osteotomy for the treatment of hip dysplasias-technique and preliminary results (reprinted from Clin Orthop, vol 232, pg 26-36, 1988). Clin Orthop Relat R. 2004;418:3-8

4. Yang S, Zusman N, Lieberman E, Goldstein RY. Developmental dysplasia of the hip. Pediatrics. 2019;143(1).

5. Albers CE, Steppacher SD, Ganz R, Tannast M, Siebenrock KA. Impingement adversely affects 10-year survivorship after periacetabular osteotomy for DDH. Clin Orthop Relat R. 2013;471(5):1602-14.

6. Clohisy JC, Ackerman J, Baca G, Baty J, Pe B, Kim YJ, et al. Patient-reported outcomes of periacetabular osteotomy from the prospective ANCHOR cohort study. J Bone Joint Surg Am. 2017;99(1):33-41.

7. Clohisy JC, Barrett SE, Gordon JE, Delgado ED, Schoenecker PL. Periacetabular osteotomy for the treatment of severe acetabular dysplasia. J Bone Joint Surg Am. 2005;87a(2):254-9.

8. Davidson D, Yen YM, Jette AM, Olarsch S, Kim YJ. Responsiveness of the SF-36 and WOMAC following periacetabular osteotomy for acetabular dysplasia. J Bone Joint Surg Am. 2011;93a(23):2214-8.

9. Jacobsen JS, Soballe K, Thorborg K, Bolvig L, Jakobsen SS, Holmich P, et al. Patient-reported outcome and muscle-tendon pain after periacetabula 
osteotomy are related: 1-year follow-up in 82 patients with hip dysplasia. Acta Orthop. 2019;90(1):40-5.

10. Livermore AT, Anderson LA, Anderson MB, Erickson JA, Peters CL. Correction of mildly dysplastic hips with periacetabular osteotomy demonstrates promising outcomes, achievement of correction goals, and excellent five-year survivorship. Bone Joint J. 2019;101 b(6):16-22.

11. Ricciardi BF, Fields KG, Wentzel C, Nawabi DH, Kelly BT, Sink EL. Complications and short-term patient outcomes of periacetabular osteotomy for symptomatic mild hip dysplasia. Hip Int. 2017;27(1):42-8.

12. Sanchez-Sotelo J, Trousdale RT, Berry DJ, Cabanela ME. Surgical treatment of developmental dysplasia of the hip in adults: I. Nonarthroplasty options. J Am Acad Orthop Surg. 2002;10(5):321-33.

13. Steppacher SD, Tannast M, Ganz R, Siebenrock KA. Mean 20-year followup of bernese periacetabular osteotomy. Clin Orthop Relat R. 2008;466(7):1633-44.

14. Trumble SJ, Mayo KA, Mast JW. The periacetabular osteotomyminimum 2 year followup in more than 100 hips. Clin Orthop Relat R. 1999:363:54-63.

15. Wasko MK, Yanik EL, Pascual-Garrido C, Clohisy JC. Psychometric properties of patient-reported outcome measures for periacetabular osteotomy. J Bone Joint Surg Am. 2019;101(6).

16. Wells J, Millis M, Kim YJ, Bulat E, Miller P, Matheney T. Survivorship of the bernese periacetabular osteotomy: what factors are associated with long-term failure? Clin Orthop Relat Res. 2017:475(2):396-405.

17. Wells J, Schoenecker P, Duncan S, Goss CW, Thomason K, Clohisy JC. Intermediate-term hip survivorship and patient-reported outcomes of periacetabular osteotomy the Washington university experience. J Bone Joint Surg Am. 2018;100(3):218-25.

18. Matheney T, Kim YJ, Zurakowski D, Matero C, Millis M. Intermediate to long-term results following the bernese periacetabular osteotomy and predictors of clinical outcome: surgical technique. J Bone Joint Surg Am. 2010;92(Suppl 1 Pt 2):115-29.

19. Griffin DR, Parsons N, Mohtadi NG, Safran MR. Multicenter Arthroscopy of the Hip Outcomes Research N. A short version of the International Hip Outcome Tool (iHOT-12) for use in routine clinical practice. Arthroscopy. 2012;28(5):611-6.

20. Gray BL, Stambough JB, Baca GR, Schoenecker PL, Clohisy JC. Comparison of contemporary periacetabular osteotomy for hip dysplasia with total hip arthroplasty for hip osteoarthritis. Bone Joint J. 2015;97(10):1322-7.

21. Rosinsky PJ, Chen JW, Yelton MJ, Lall AC, Maldonado DR, Meghpara MB, et al. Does failure to meet threshold scores for $\mathrm{mHHS}$ and iHOT-12 correlate to secondary operations following hip arthroscopy? J Hip Preserv Surg. 2020;7(2):272-80.

22. Kraeutler MJ, Safran MR, Scillia AJ, Ayeni OR, Garabekyan T, Mei-Dan O. A Contemporary look at the evaluation and treatment of adult borderline and frank hip dysplasia. Am J Sport Med. 2020;48(9):2314-23.

23. Nepple JJ, Wells J, Ross JR, Bedi A, Schoenecker PL, Clohisy JC. Three patterns of acetabular deficiency are common in young adult patients with acetabular dysplasia. Clin Orthop Relat R. 2017;475(4):1037-44.

24. Yang YH, Zuo JL, Liu T, Xiao JL, Liu SL, Gao ZL. Morphological analysis of true acetabulum in hip dysplasia (crowe classes I-IV) via 3-D implantation simulation. J Bone Joint Surg Am. 2017;99(17).

25. Kalore NV, Jiranek WA. Save the torn labrum in hips with borderline acetabular coverage. Clin Orthop Relat R. 2012;470(12):3406-13.

26. Larson CM, Moreau-Gaudry A, Kelly BT, Byrd JWT, Tonetti J, Lavallee $\mathrm{S}$, et al. Are normal hips being labeled as pathologic? A CT-based method for defining normal acetabular coverage. Clin Orthop Relat R. 2015;473(4):1247-54

27. Kovalenko B, Bremjit P, Fernando N. Classifications in brief: tonnis classification of hip osteoarthritis. Clin Orthop Relat R. 2018;476(8):1680-4.

28. Tonnis $D$, Heinecke A. Acetabular and femoral anteversion: relationship with osteoarthritis of the hip. J Bone Joint Surg Am. 1999;81a(12):1747-70

29. Yasunaga Y, Ochi M, Terayama $H$, Tanaka R, Yamasaki T, Ishii Y. Rotational acetabular osteotomy for advanced osteoarthritis secondary to dysplasia of the hip. J Bone Joint Surg Am. 2006;88a(9):1915-9.

30. Chang CH, Chiang YT, Lee ZL, Kuo KN. Incidence of surgery in developmental dysplasia of the hip in Taiwan. J Formos Med Assoc. 2007;106(6):462-6.

31. Furnes O, Lie SA, Espehaug B, Vollset SE, Engesaeter LB, Havelin LI. Hip disease and the prognosis of total hip replacements-a review of 53698 primary total hip replacements reported to the Norwegian Arthroplasty Register 1987-99. J Bone Jt Surg Br. 2001;83(4):579-86.

32. Dindo D, Demartines N, Clavien PA. Classification of surgical complications - a new proposal with evaluation in a cohort of 6336 patients and results of a survey. Ann Surg. 2004;240(2):205-13.

33. McAuley JP, Szuszczewicz ES, Young A, Engh CA Sr. Total hip arthroplasty in patients 50 years and younger. Clin Orthop Relat Res. 2004;418:119-25.

34. Matheney T, Kim YJ, Zurakowski D, Matero C, Millis M. Intermediate to long-term results following the bernese periacetabular osteotomy and predictors of clinical outcome. J Bone Joint Surg Am. 2009;91a(9):2113-23.

35. Lerch TD, Steppacher SD, Liechti EF, Tannast M, Siebenrock KA. One-third of hips after periacetabular osteotomy survive 30 years with good clinical results, no progression of arthritis, or conversion to THA. Clin Orthop Relat Res. 2017;475(4):1154-68.

36. Schmaranzer F, Haefeli PC, Hanke MS, Liechti EF, Werlen SF, Siebenrock KA et al. How does the dGEMRIC index change after surgical treatment for FAl? A prospective controlled study: preliminary results. Clin Orthop Relat Res. 2017;475(4):1080-99.

\section{Publisher's Note}

Springer Nature remains neutral with regard to jurisdictional claims in published maps and institutional affiliations.
Ready to submit your research? Choose BMC and benefit from:

- fast, convenient online submission

- thorough peer review by experienced researchers in your field

- rapid publication on acceptance

- support for research data, including large and complex data types

- gold Open Access which fosters wider collaboration and increased citations

- maximum visibility for your research: over $100 \mathrm{M}$ website views per year

At BMC, research is always in progress.

Learn more biomedcentral.com/submissions 